\title{
Erratum to: short-term efficacy of intravitreal Aflibercept injections for retinal angiomatous proliferation
}

Hung-Da Chou', Wei-Chi Wu ${ }^{1,2}$, Nan-Kai Wang ${ }^{1,2}$, Lan-Hsin Chuang ${ }^{2,3}$, Kuan-Jen Chen ${ }^{1,2}$ and Chi-Chun Lai ${ }^{1,2^{*}}$

\section{Erratum}

After publication of the article [1], it has been brought to our attention that Table 1 has been formatted incorrectly in the PDF version. The table should be clearly divided into two groups of patients. Patients $1-11$ are in the "Naïve Group" and patients 12-19 are in the "Pre-treatment Group". This has been updated to reflect this in the original version of the article.

\section{Author details}

'Department of Ophthalmology, Chang Gung Memorial Hospital, Linkou, No. 5, Fuxing St., Guishan Dist, Taoyuan City 333, Taiwan, Republic of China. ${ }^{2}$ School of Medicine, Chang Gung University, No. 259, Wenhua 1st Rd., Guishan Dist, Taoyuan City 333, Taiwan, Republic of China. ${ }^{3}$ Department of Ophthalmology, Chang Gung Memorial Hospital, Keelung, No. 222, Maijin Rd., Anle Dist, Keelung City 204, Taiwan, Republic of China.

Received: 24 July 2017 Accepted: 25 July 2017

Published online: 15 August 2017

\section{Reference}

1. Chou H, Wu W, Wang N, Chuang L, Chen K, Lai C. Short-term efficacy of intravitreal Aflibercept injections for retinal angiomatous proliferation. BMC Ophthalmology. 2017;17:1. doi:10.1186/s12886-017-0497-0.

\footnotetext{
* Correspondence:

'Department of Ophthalmology, Chang Gung Memorial Hospital, Linkou, No. 5, Fuxing St., Guishan Dist, Taoyuan City 333, Taiwan, Republic of China ${ }^{2}$ School of Medicine, Chang Gung University, No. 259, Wenhua 1st Rd., Guishan Dist, Taoyuan City 333, Taiwan, Republic of China
} 